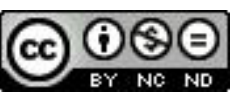

\title{
Sequência Didática para o Ensino de Trigonometria Usando o Software GeoGebra
}

\section{Didactic Sequence for Teaching Trigonometry Using the Software GeoGebra}

Maria Maroni Lopes*

\begin{abstract}
Resumo
Este artigo visa a apresentação de um produto educacional, um caderno de atividades com recomendação de uso para sala de aula do ensino fundamental e médio, que teve como argumentos teóricos as investigações realizadas na dissertação de Mestrado Profissional em ensino de Matemática. Objetivamos, com esse estudo, analisar algumas das potencialidades e limitações do software GeoGebra no ensino e na aprendizagem de trigonometria. Adotamos as concepções da Didática da Matemática no que se refere ao uso das Tecnologias de Informação e Comunicação com os recursos de um software de geometria dinâmica e atividades investigativas. Para tanto, descrevemos brevemente a estrutura do caderno de atividades, apresentando as teorias que o fundamentaram e os objetivos que nortearam sua elaboração.
\end{abstract}

Palavras-chave: Produto Educacional. Software de Geometria Dinâmica. Atividades Investigativas.

\begin{abstract}
This article describes a workbook of educational activities recommended for use in elementary and high school classrooms. It is based on theoretical arguments resulting from investigations carried out in the Professional Master's degree program in teaching of mathematics. We aimed to analyze some of the capabilities and limitations of the

\footnotetext{
* Mestre em Ensino de Ciências Naturais e Matemática pela Universidade Federal do Rio Grande do Norte (UFRN). Professora do Centro de Ciências Exatas e Aplicadas da Universidade Federal do Rio Grande do Norte, Caicó, RN, Brasil. Endereço para correspondências: Rua Arnaldo Neves Silva, Bloco 12, Apto. 103, Neópolis, CEP: 59080-460, Natal, RN, Brasil. E-mail: marolopes@ hotmail.com.
} 
software GeoGebra in the teaching and learning of trigonometry. We adopted the concepts of the didactic of mathematics related to the use of Information and Communication Technologies with the resources of dynamic geometry software and investigative activities. We briefly describe the structure of the activities workbook, presenting the theories on which it was based and the objectives that guided its elaboration.

Keywords: Educational Product. Dynamic Geometry Software. Investigative Activities.

\section{Introdução}

Apresentamos e discutimos, neste artigo, aspectos relativos ao produto educacional elaborado no mestrado profissional em Ensino de Ciências Naturais e Matemática da Universidade Federal do Rio Grande do Norte (UFRN).

A elaboração do produto educacional é um processo reflexivo e contextualizado, que contém tanto alguns dos saberes da experiência docente dos professores quanto a teoria desenvolvida por pesquisadores e estudadas com profundidade. Esse produto não é um material didático pronto para ser utilizado por professores e estudantes, mas um conjunto de orientações de atividades que podem ser adaptadas pelos professores em suas salas de aula de acordo com as turmas com as quais estejam atuando.

O nosso produto educacional, apresentado neste artigo, traz como argumento teórico os estudos realizados na elaboração da nossa dissertação de mestrado, desenvolvida junto ao Programa de Pós-Graduação em Ensino de Ciências Naturais e Matemática (PPGCNM) da Universidade Federal do Rio Grande do Norte (UFRN) que tem como título: Construção e aplicação de uma sequência didática para o ensino de trigonometria usando o software GeoGebra ${ }^{1}$. O principal objetivo da nossa pesquisa foi analisar algumas das potencialidades e limitações do software GeoGebra no ensino e na aprendizagem de Trigonometria, e elaborar um Caderno de atividades com recomendações de uso para sala de aula do Ensino Fundamental e Médio.

\section{Princípios norteadores: tecnologias de informação e comunicação (TIC), geometria dinâmica e atividades investigativas}

A presença da linguagem digital, expressa em múltiplas TIC, vem impondo

\footnotetext{
${ }^{1}$ É um software dinâmico, que reúne Geometria, Álgebra, Cálculo e Estatística. Permite a construção de vários objetos como: pontos; vetores; segmentos; retas; secções cônicas; gráficos de funções e curvas parametrizadas, os quais podem, depois, serem modificados dinamicamente. Permite, ainda, a introdução de equações e coordenadas, digitando-se diretamente na sua caixa de entrada. O software apresenta três diferentes janelas: gráfica, algébrica ou numérica, e a folha de cálculo.
} 
mudanças no modo como obtemos informação e nos comunicamos, e a chegada desses recursos na escola nos faz refletir sobre seu uso em sala de aula, analisando de que forma essas ferramentas podem contribuir para uma formação do aluno compatível com os avanços proporcionado pela sociedade da informação. "A inserção da tecnologia na educação deve ser compreendida e orientada no sentido de proporcionar nos indivíduos o desenvolvimento de uma inteligência crítica, mais livre e criadora" (MISKULIN, 2008, p. 219).

Assim, o poder da linguagem digital, baseado no acesso a computadores e a todos os seus periféricos, à internet, e aos software tem influenciado, cada vez mais, a forma como construímos nossos conhecimentos e como a escola organiza seus currículos. Para Kenski (2009), quando esses recursos são bem utilizados provocam alterações nas relações entre professores e alunos e, ainda, proporcionam um maior aprofundamento nos conteúdos estudados.

Assim sendo, o processo de ensino e de aprendizagem permeado pelas Tecnologias de Informação e Comunicação está intimamente ligado à abordagem pedagógica adotada. Ponte (2003) destaca a relevância dos professores de Matemática, em sua prática, dominarem as ferramentas das TIC em suas salas de aula, incluindo software educacionais próprios da sua disciplina ou de educação no âmbito geral.

Essas ferramentas são consideradas por Kenski (2009) não apenas um suporte tecnológico nas atividades de sala de aula, elas interferem em nossa forma de pensar, de nos relacionarmos, de adquirirmos conhecimentos. A autora destaca, ainda, que meios de comunicação como a televisão e o computador, a partir de seus recursos, movimentaram a educação e provocaram novas mediações entre a abordagem do professor, a compreensão do aluno e o conteúdo que circula nesses meios.

Pesquisas que analisam as potencialidades das TIC em sala de aula ressaltam a sua relevância no ensino de Matemática. Borba e Penteado (2005) e Scheffer (2002) asseguram que as TIC podem ser grandes aliadas no ensino da Matemática, visto que permitem a experimentação e a ênfase no processo de visualização. Ao incluir os recursos da informática como parte das atividades em sala de aula, tem-se a possibilidade de o aluno realizar descobertas, incentivando a compreensão e dando significado ao conhecimento matemático.

Assim sendo, quando a informática faz parte do ambiente escolar, num processo dinâmico de interação entre alunos, professores e TIC, ela passa a despertar no professor a sensibilidade para as diferentes possibilidades de representação da Matemática, o que é importante no momento de realizar 
construções, análises, observações de regularidades e, também, ao estabelecer relações. "Trabalhar a informática na escola na perspectiva de produzir conhecimentos permite ao aluno fazer análises de modo a poder refletir sobre seus procedimentos de solução, testes e conceitos empregados na resolução de problemas" (SCHEFFER, 2002, p. 23).

Nesse sentido, Kaput e Thompson (1994 apud SCHEFFER, 2002), quando se referem à pesquisa com tecnologias na Educação Matemática, destacam três aspectos que podem promover uma profunda transformação na experiência de fazer e aprender matemática: Interatividade - proporciona a interação entre o homem e o saber produzido; Controle utilizável dos ambientes de aprendizagem - favorece a resolução de problemas, e Conectividade que possibilita a conexão entre professores e alunos de diferentes partes do mundo.

Segundo Valente (1999), existem formas diferenciadas de se trabalhar com o computador na educação. Por exemplo, as atividades de uso do computador podem servir para transmitir informação ao aluno, e consiste na informatização dos tradicionais métodos de ensino. Nesse caso, o professor está apenas mudando de mídia, saindo do quadro e giz para o computador. Outra prática diz respeito a quando o aluno usa o computador para construir seus conhecimentos, situação que favorece a interação do aluno com objetos do ambiente computacional. $\mathrm{O}$ computador passa a ser uma máquina para ser ensinada, propiciando condições para o aluno descrever a resolução de problemas, refletir sobre os resultados obtidos e depurar ${ }^{2}$ suas ideias por intermédio da busca de novos conteúdos e novas estratégias.

Ainda, segundo Valente (1999), o envolvimento com o objeto em construção cria oportunidades para o aluno colocar em prática alguns dos conhecimentos matemáticos que possui. Se esses conhecimentos não são suficientes para resolver os problemas encontrados, o aluno terá que buscar novas informações nas mais variadas fontes às quais tenha acesso.

Desse modo, o aluno usa o computador para resolver problemas, ou seja, realizar tarefas como desenhar, escrever, construir, calcular, analisar, após efetuar alguns comandos, levantar hipóteses, formular e testar conjeturas, entre outras possibilidades. A construção do conhecimento advém do fato de o aluno ter de buscar novos conteúdos e estratégias para acrescer ao conhecimento de que já dispõe sobre o assunto, que está sendo estudado via computador.

Diante desse contexto, recorremos, em nosso estudo, às ferramentas

${ }^{2}$ Processo de encontrar e reduzir defeitos num aplicativo de software ou mesmo em hardware. 
das TIC por meio dos recursos de um software de Geometria Dinâmica, em específico o software GeoGebra. Para tanto, nos baseamos em autores como Gravina (1996) e Zulatto (2002) que nos mostram que, ambientes de Geometria Dinâmica podem ser ferramentas riquíssimas na superação das dificuldades dos alunos inerentes ao processo de ensino e de aprendizagem de conteúdos matemáticos. Conforme Zulatto (2002), com o recurso de um software de Geometria Dinâmica os alunos podem realizar construções que, usualmente, fazem com régua e compasso, instrumentos que não lhes permite interagir com o desenho, por serem estáticos.

Assim sendo, o que difere numa atividade com o recurso do software é a possibilidade de movimentação dos objetos e, a partir desses movimentos, o aluno investigar o que acontece com a sua construção, levantando hipóteses como: a construção permanece com as mesmas características? Um simples movimento muda todas as características originais? Entre várias hipóteses que são possíveis levantar diante das próprias tomadas de decisão, percebendo assim as suas regularidades.

Conforme Goldenberg e Cuoco (1998), a expressão Geometria Dinâmica foi inicialmente usada por Nick Jackiw e Steve Rasmussem, de forma genérica, com o objetivo de apresentar a diferença entre software de Geometria Dinâmica e outros software de Geometria. Os software de Geometria Dinâmica possuem um recurso que possibilita a transformação contínua em tempo real, ocasionada pelo arrastar (GODENBERG; CUOCO, 1998, p. 132).

De modo geral, uma das principais características de um software de Geometria Dinâmica é a possibilidade de movimentar os objetos na tela sem alterar as propriedades da construção inicial, com isso, tem-se a possibilidade de, numa atividade desenvolvida com os recursos de um software com essas características, se fazer investigações, descobertas, confirmar resultados e fazer simulações, permitindo, inclusive, levantar questões relacionadas com a sua aplicação prática.

Gravina (1996) destaca o potencial das múltiplas representações em um software de Geometria Dinâmica, considerando que um mesmo objeto matemático pode receber diferentes representações, as quais registram diferentes facetas do mesmo, uma exploração que transita em diferentes sistemas torna-se significativa no processo de construção do conceito. Como exemplo, podemos destacar uma construção realizada com o software GeoGebra que permite mostrar os objetos matemáticos em três diferentes representações: graficamente (pontos, gráficos de funções), algebricamente (coordenadas de pontos, equações) e nas 
células da folha de cálculo. Assim, todas as representações do mesmo objeto estão ligadas dinamicamente e adaptam-se automaticamente às mudanças realizadas em quaisquer delas, independentemente da forma como esses objetos foram inicialmente construídos.

Contudo, para a elaboração das atividades da sequência didática presente no produto educacional, baseadas nos recursos das TIC por meio das ferramentas do software GeoGebra, adotamos uma perspectiva investigativa, estabelecendo um diálogo constante entre as investigações no ensino de Matemática e os recursos das TIC em sala de aula.

O termo investigação apresenta diferentes significados, podendo ser aplicado nos mais variados contextos. De acordo com Ponte, Brocardo e Oliveira (2005, p. 13), “[...] para os matemáticos profissionais, investigar é descobrir relações entre objetos matemáticos conhecidos ou desconhecidos, procurando identificar as respectivas propriedades".

Assim, ao definirem investigação, os autores supracitados apontam que, nesse tipo de atividade, o aluno se vê envolvido em um ou mais problemas. $\mathrm{O}$ primeiro momento de uma investigação se dá na descoberta do problema, ou seja, na sua identificação, é preciso haver clareza do que realmente será investigado para que, em seguida, seja possível determinar as estratégias de resolução. Ainda, segundo esses autores, investigar em matemática é descobrir relações entre objetos matemáticos conhecidos ou desconhecidos, procurando identificar as suas propriedades.

Em Brocardo (2001) encontramos uma observação de Pirie, ressaltando que uma investigação constitui uma situação aberta, uma exploração que não tem como objetivo chegar a uma resposta certa. Pelo contrário, o objetivo é o caminho a ser seguido e não o resultado. Ao propor aos alunos uma investigação, pretende-se que eles explorem possibilidades, formulem conjeturas e confiem na validade de suas descobertas.

As investigações matemáticas podem apresentar um grande potencial educativo, mostrando-se importantes no desenvolvimento da criatividade do aluno. Segundo Brocardo (2001), uma investigação matemática é uma atividade que envolve três processos: Exploração de Possibilidades; Formulação de conjecturas; Argumentos que validem as hipóteses levantadas. Nessa perspectiva, as atividades de investigação são caracterizadas por vários processos matemáticos que não podem ser seguidos de forma linear, visto que, ao perceber que os testes realizados não confirmam determinadas conjeturas, é necessário voltar atrás, reavaliar o que está sendo feito, e formular novas 
conjeturas. Para isso, é preciso visualizar o que ocorreu para que a primeira conjetura não se sustentasse ou o que houve para que o caminho fosse alterado. Portanto uma atividade de investigação permite que o aluno reinicie os questionamentos quantas vezes forem necessárias.

Ponte e Matos (1996) afirmam que numa atividade de investigação matemática os alunos são colocados frente a frente com questões que sugerem o levantamento de hipóteses, a elaboração de conjeturas, o teste das hipóteses e a busca de falhas de abordagem ou apoio de fundamentos que as sustentem.

Nesse sentido, investigar nada mais é do que buscar, procurar conhecer, tentar elaborar soluções, descobrir padrões, relações, semelhanças e diferenças, de forma a conseguir chegar a generalizações.

\section{Estrutura do nosso produto educacional}

O produto educacional aqui exposto traz, inicialmente, uma descrição rápida do software GeoGebra, apresentando sua tela principal, algumas de suas ferramentas e comandos que são utilizados na construção de figuras, objetivando, com isso, que os professores se familiarizem com o software.

Na segunda parte, apresenta-se uma sequência de seis atividades sobre o conteúdo de trigonometria por meio dos recursos do software GeoGebra. As atividades foram elaboradas numa perspectiva investigativa, tomando como referência as concepções de Ponte, Brocardo, Oliveira (2005).

\section{Contexto e desenvolvimento do produto educacional}

A construção da nossa sequência de atividades, presentes no produto educacional, teve como ponto de partida o objetivo geral da nossa dissertação de mestrado: analisar as potencialidades e limitações do software GeoGebra no ensino e aprendizagem de trigonometria.

Os dados utilizados em nossa dissertação de mestrado, a qual motivou a elaboração do produto educacional, foram coletados com alunos da segunda série do Ensino Médio do turno matutino de uma escola pública do Estado do Rio Grande do Norte, na cidade de Natal. A turma ${ }^{3}$ contava com 42 alunos, sendo que a frequência média era de 34 alunos. Iniciamos nossas atividades em

\footnotetext{
${ }^{3}$ A professora pesquisadora atuava nessa turma como bolsista REUNI (Reestruturação e Expansão das Universidades Federais) juntamente com alunos da Licenciatura em Matemática (bolsistas PIBID - Programa Institucional de Bolsas de Iniciação à Docência).
} 
março de 2010 e encerramos no início de maio de 2010. Ao todo, foram 15 encontros de 50 minutos cada.

Para atingir o propósito do nosso estudo, o procedimento de coleta de dados se deu por meio de um questionário sobre o conhecimento que os alunos tinham em relação às ferramentas das Tecnologias de Informação e Comunicação; entrevista após o desenvolvimento das atividades e análise descritiva das atividades.

Durante o desenvolvimento das atividades, os alunos receberam uma apostila com o roteiro de cada uma delas. Trabalharam em dupla durante os 15 encontros, que aconteceram no laboratório de informática ${ }^{4}$.

Percebemos que as discussões entre as duplas fluíram significativamente, os alunos passaram a ler os questionamentos apresentados no roteiro das atividades e a discutirem entre si, analisando cada passo das construções, levantando hipóteses, fazendo análises e argumentando sobre suas conclusões. O exercício de construir triângulos semelhantes, por exemplo, valendo-se do movimento de arrastar um dos vértices, promoveu a discussão que, em decorrência, permitiu que uma argumentação mais consistente sobre os resultados fosse elaborada. Os alunos perceberam a possibilidade de criar triângulos semelhantes traçando uma reta paralela a uma das bases do triângulo.

\section{Descrição das atividades presentes no produto educacional}

O produto educacional foi elaborado com base nas atividades desenvolvidas com os alunos do $2^{\circ}$ série do ensino médio, conforme mencionado anteriormente. Foram realizadas seis atividades divididas em dois blocos. As atividades do bloco um referiam-se, mais especificamente, às dificuldades dos alunos em relação a conteúdos base para o estudo de trigonometria, por exemplo, questões relacionadas à geometria. Tendo como referência as concepções de Brito e Morey (2004, p. 65-70), em estudo realizado com professores da rede pública do RN, que enfatizam as dificuldades que esses professores encontravam no ensino de trigonometria por falta de conhecimento de conceitos básicos de geometria.

Além disso, o bloco um continha atividades de familiarização do software GeoGebra. As primeiras atividades pertencentes ao bloco um, referem-se a:

\footnotetext{
${ }^{1}$ O laboratório da escola contava com apenas 10 computadores. Para melhor desenvolvimento de nossas atividades dividíamos a turma em dois grupos, para isso, contávamos com a ajuda de dois bolsistas PIBID. Um bolsista ficava com parte da turma em sala de aula e o outro bolsista nos ajudava com o grupo de alunos que levávamos ao laboratório de informática.
} 
soma dos ângulos internos de um triângulo; altura de triângulos e semelhança de triângulos.

As atividades do bloco dois referem-se a: Razões trigonométricas nos triângulos retângulos; Ciclo trigonométrico e Funções trigonométricas com ênfase nas funções seno, cosseno e tangente.

Atividade 01: Soma dos ângulos internos de um triângulo

Procedimentos: construa um triângulo de vértices A, B e C (selecione o botão polígono $\mathrm{D}$ e clique na janela gráfica); determine a amplitude dos ângulos (clique no botão um dos vértices do triângulo $\mathbf{A B C}$, o que acontece com a soma dos ângulos? Atividade 02: Altura de triângulos

Objetivos: familiarizar os alunos com o software GeoGebra; perceber o segmento altura com mais significado, desmistificando algumas ideias que parte dos alunos apresenta em relação à altura de um triângulo percebendo como um segmento interno ao triângulo.

Procedimentos: trace uma reta $A B$; marque um ponto $C$ fora da reta; trace uma reta paralela a reta $\mathrm{AB}$ passando por $\mathrm{C}$; marque um ponto $\mathrm{D}$ na reta $b$ paralela a reta $\mathrm{AB}$; trace uma reta perpendicular a reta $\mathrm{AB}$ passando por $\mathrm{D}$; construa um triângulo ligando os pontos $\mathrm{A}, \mathrm{B}, \mathrm{D}$ e $\mathrm{A}$. Faça o vértice $D$ do triângulo deslocar-se na reta paralela ao lado $\mathrm{AB}$ do triângulo; o que você observa ao movimentar o vértice $\mathrm{D}$ ? Justifique. Agora determine a área do triângulo $\mathrm{ABD}$, movimente o vértice $D$ do triângulo na reta $\mathrm{b}$. O que você observa? Atividade 03: Semelhança de triângulos

Objetivos: familiarização dos alunos com o software GeoGebra; investigar propriedades em triângulos semelhantes; perceber que os triângulos retângulos não são sempre semelhantes.

Procedimentos: construa um triângulo de vértices A, B e C (selecione o botão polígono e clique na janela gráfica); marque um ponto $D$ no lado $A B$ do triângulo; trace uma reta paralela ao lado $B C$ (clique no botão E, no lado $\boldsymbol{B C}$ e no ponto $\boldsymbol{D}$ ); marque a intersecção entre a reta e o lado do triângulo $A B C$ ( clique no botão no lado e na reta); trace o novo triângulo ligando os pontos $A, D, E$; determine o comprimento dos lados (clique 
no botão [m e nos lados dos triângulos) e a amplitude dos ângulos dos triângulos $A B C$ e $A D E$ ( clique no botão 2 e nos triângulos); encontre a razão entre os lados dos triângulos $A B C$ e $A D E$ (digite na caixa de entrada al $b$, por exemplo, aparecerá na janela algébrica o valor correspondente); movimente um dos vértices do triângulo $A B C$. O que acontece com a razão entre lados? Movimente o ponto $D$ vértice do triângulo $A D E$, o que acontece com a razão entre os lados? Que conclusões você chegou em relação aos triângulos $\mathrm{ABC}$ e $\mathrm{ADE}$ ?

Atividade 04: Razões Trigonométricas nos triângulos retângulos.

Objetivos: familiarizar os alunos com o software GeoGebra; investigar as propriedades, noções e conceitos das razões trigonométricas nos triângulos retângulos.

Procedimentos: trace um segmento de reta $A B$ (clique no botão e na janela gráfica); trace uma reta $b$ perpendicular ao segmento $A B$ passando por A (clique no botão $\forall$ e em seguida no segmento $A B$ e no ponto A); marque um ponto $\mathrm{C}$ sobre a reta $\mathrm{b}$, (clique no botão e na reta); construa $\mathrm{o}$ triângulo (clique no botão polígono da barra de ferramenta $\$$ e em seguida nos pontos $A, B, C$ e $A$ ); determine o comprimento dos lados e a amplitude dos ângulos; encontre a razão entre os lados do triângulo, digite na caixa de entrada (distância [A, C]/distância [B,C]) em seguida digite (distância $[A, B] /$ distância $[B, C])$, aparecerá na janela algébrica a razão entre os lados do triângulo. Arraste o vértice $B$ do triângulo, o que você observa em relação às razões? Justifique suas conclusões.

Atividade 05: Ciclo Trigonométrico

Objetivos: familiarizar os alunos com o software GeoGebra; investigar as propriedades, noções e conceitos das razões trigonométricas no ciclo trigonométrico.

Procedimentos: construa uma circunferência com centro em A $(0,0)$ passando por $\mathrm{B}(0,1)$, (Clique em exibir eixo). Insira o ponto $\mathrm{A}$ Entrada: $\quad A=(0,0)$ na caixa de entrada e em seguida tecle enter, insira o ponto $\mathrm{B}=(1,0)$. Fixe os pontos A e B (clique em cima dos pontos com o botão direito do mouse aparecerá a seguinte caixa de diálogo 


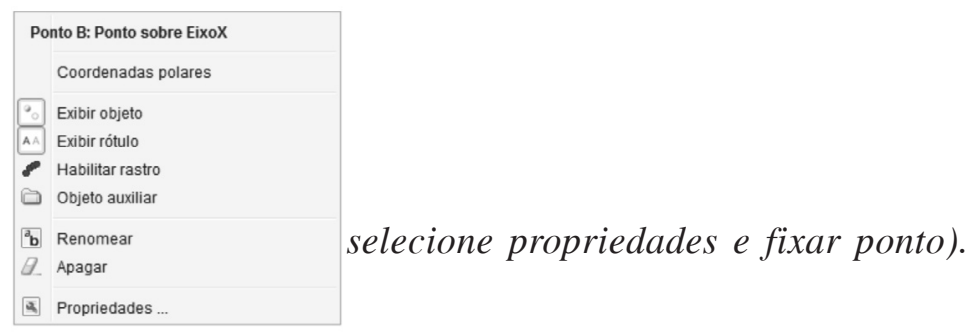

Selecione o botão $\odot$ e clique nos pontos A e B; marque um ponto C na circunferência no primeiro quadrante, trace uma reta perpendicular ao segmento $\mathrm{AB}$ passando por $\mathrm{C}$; assinale a intersecção entre a reta $r$ e o segmento $\mathrm{AB}$ (selecione o botão $\mathrm{D}$ e clique no segmento $\mathrm{AB}$ e no ponto $C$ ); trace o triângulo ACD (clique no botão Do nos pontos $A, C, D$ e A); utilizando a ferramenta distância, determine a medida dos lados do triangulo ACD e complete a tabela.

\begin{tabular}{|l|l|l|l|}
\hline Medida de AD & Medida de DC & Medida de AC & DC/AC \\
\hline & & & \\
\hline & & & \\
\hline
\end{tabular}

Arraste o ponto $\mathrm{C}$ duas vezes e anote as medidas na tabela, você percebe alguma propriedade? Discuta com seus colegas e anote as observações feitas. Atividade 06: Funções Trigonométricas

Objetivos: familiarizar os alunos com o software GeoGebra; investigar os efeitos dos parâmetros $a, b, c$ e $d$ em casos particulares das funções: seno, cosseno e tangente.

Procedimento: digite na caixa de entrada a função $f(x)=\sin (x)$, observe o gráfico. Digite na mesma janela gráfica, as seguintes funções: $g(x)=2 * \sin (x)$; $h(x)=3 * \sin (\mathrm{x})$ e $\mathrm{p}(\mathrm{x})=5 * \sin (\mathrm{x})$. Analise o que pode ser observado em relação a imagem da função. Discuta com seus colegas e justifique as conclusões que vocês chegaram.

Agora digite as funções: $f(x)=\sin (x) ; g(x)=-2 * \sin (x) ; h(x)=-$ $3 * \sin (x)$ e $t(x)=-4 * \sin (x)$ na mesma janela gráfica. Verifique o comportamento das funções em relação a função $f(x)=\sin (x)$, que conclusões você chegou em relação a imagem dessas funções? 
Digite na caixa de entrada inicialmente a função $f(x)=\sin (x)$ em seguida digite as seguintes funções: $g(x)=\sin (2 x) ; h(x)=\sin (3 x) ; t(x)=\sin (4 x)$. Qual o comportamento dessas funções em relação a função $f(x)=\sin (x)$ ? $\mathrm{O}$ que pode perceber em relação a imagem dessas funções?

Insira na caixa de entrada a função $f(x)=a^{*} \sin \left(b^{*} x+c\right)+d$ ( para isso, insira inicialmente os parâmetros $a, b, c, e d)$. Movendo o seletor a o que pode ser percebido no gráfico de f? Proceda de modo análogo com os demais parâmetros. O que você observou? Discuta com seus colegas o comportamento do gráfico após movimentar os parâmetros. Justifique suas conclusões.

Agora é com você, proceda de maneira análoga para as funções: cosseno e tangente.

\section{Divulgação do produto}

O produto educacional intitulado Sequência Didática para o Ensino de Trigonometria usando o software GeoGebra encontra-se no site do PPGECN $^{5}$, na secretaria do referido programa, no site da Biblioteca Central ${ }^{6}$ da UFRN e nos anais do III Encontro Regional de Educação Matemática do $\mathrm{RN}-\mathrm{EREM}^{7}$.

\section{Considerações finais}

O produto educacional, um caderno de atividades com sugestões de uso para sala de aula de matemática, foi desenvolvido pela professora pesquisadora sobre a orientação da Professora Bernadete Barbosa Morey. Desse caderno constam atividades referentes ao conteúdo de trigonometria.

Concluímos, com base nos resultados de nossa pesquisa, que, dentre as potencialidades apresentadas pelo software GeoGebra no ensino e na aprendizagem de trigonometria por meio de atividades investigativas estão, principalmente, a construção, o dinamismo, a investigação, visualização e argumentação.

Contudo, à medida que os parâmetros do software são manipulados consegue-se visualizar as alterações realizadas nas suas construções e fazer

\footnotetext{
${ }^{5}$ Disponível em: <http://www.sigaa.ufrn.br/sigaa/public/programa/portal.jsf?lc=pt_BR\&id=134>.

${ }^{6}$ Disponível em: <http://www.bczm.ufrn.br/site/>.

${ }^{7}$ Disponível em: <http://www.sbemrn.com.br/site/III\%20erem/minicurso/doc/MC_Lopes.pdf>.
} 
inferências sobre as mesmas. Borba e Villarreal (2005) apontam que a visualização em Matemática está vinculada a habilidades de interpretar e manipular imagens figurais. Nesse sentido, destacamos que a visualização foi um ponto forte no levantamento de hipóteses e formulação de conjeturas a partir da análise de construções produzidas em nosso estudo.

Em relação às limitações de uso do software, evidenciamos algumas dificuldades de ordem estrutural, como por exemplo: poucos computadores para turmas muito grandes e a falta de conhecimento, pelos professores, do sistema operacional instalado nas escolas.

Assim sendo, esperamos que o caderno de atividades de trigonometria com os recursos do software GeoGebra, presentes em nosso produto educacional, possa contribuir com a prática dos professores possibilitando a adequação do material às turmas com as quais estejam atuando.

\section{Referências}

BORBA, M. C.; PENTEADO M. G. Informática e educação matemática. Belo Horizonte: Autêntica, 2005.

BORBA, M. C.; VILLARREAL, M. E. Humans-with-media and the reorganization of mathematical thinking: information and communication technologies, modeling, experimentation and visualization. New York: Springer, 2005. (Mathematics Education Library, 39).

BRITO, A. J.; MOREY, B. B. Trigonometria: dificuldades dos professores de Matemática do ensino fundamental. Revista Horizontes, Bragança Paulista, v. 22, n. 1, p. 65-70, jan./jun. 2004.

BROCARDO J. As Investigações na aula de Matemática: um projeto curricular no $8^{\circ}$ ano. 2001. 200f. Tese (Doutorado em Educação Matemática) - Universidade de Lisboa, Lisboa, 2001.

GOLDENBERG, E. P. e CUOCO, A. A. What is dynamic geometry? In: LEHER, R.; CHAZAN, D. (Ed.). Designing learning environments for developing urderstanding of geometry and space. London: Lawrence Erlbaum Associates, 1998. p. 350-367.

GRAVINA, M. A. Geometria dinâmica: uma nova abordagem para o aprendizado da Geometria. In: SIMPÓSIO BRASILEIRO DE INFORMÁTICA NA EDUCAÇÃO, 7., 1996, Belo Horizonte. Anais... Belo Horizonte: SBC, 1996. p. 1-13. CD-ROM 
KENSKI, V. M. Educação e tecnologias: o novo ritmo da informação. 5. ed. Campinas: Papirus, 2009.

MISKULIN, R. G. S. As possibilidades didático-pedagógicas de ambientes computacionais na formação colaborativa de professores de matemática. In: FIORENTINI, D. (Org.). Formação de professores de matemática: explorando novos caminhos com outros olhares. Campinas: Mercado de letras, 2008. p. 217-248.

PONTE J. P. Investigação sobre investigações matemáticas em Portugal. Investigar em Educação, Lisboa, 2003. p. 1-75. Disponível em: <http://www.fc.ul.pt/docentes/ jponte/artigo/_pt.htm>. Acesso em: 10 out. 2008.

PONTE J. P.; BROCARDO, J.; OLIVEIRA, H. Investigações matemáticas na sala de aula. Belo Horizonte: Autêntica, 2005.

PONTE, J. P.; MATOS, J. F. Processos cognitivos e interacções sociais nas investigações matemáticas. In: ABRANTES, P.; LEAL, L. C.; PONTE, J. P. (Ed.). Investigar para aprender matemática. Lisboa: Projecto MPT e APM, 1996. p. 119-138.

SCHEFFER, N. F. Corpo tecnologias matemática: uma interação possível no ensino fundamental. Erechim: Edifapes, 2002.

VALENTE, J. A. (Org.). O computador na sociedade do conhecimento. Campinas: UNICAMP/NIED, 1999.

ZULATTO, R. B. A. Professores de matemática que utilizam softwares de geometria dinâmica: suas características e perspectivas. 2002. 184f. Dissertação (Mestrado em Educação Matemática) - Instituto de Geociências e Ciências Exatas, Universidade Estadual Paulista, Rio Claro, 2002.

Submetido em Agosto de 2012. Aprovado em Novembro de 2012. 